\title{
TIPOS, CAUSAS E ESTRATÉGIAS DE INTERVENÇÃO FRENTE A ERROS DE MEDICAÇÃO: UMA REVISÃO INTEGRATIVA
}

\section{TYPES, CAUSES AND INTERVENTION STRATEGIES FACING MEDICATION ERRORS: AN INTEGRATIVE REVIEW}

\section{TIPOS, CAUSAS Y ESTRATEGIAS DE INTERVENCIÓN CARA A ERRORES DE MEDICACIÓN: UNA REVISIÓN INTEGRADORA}

\begin{abstract}
Talita Wérica Borges Figueiredo ${ }^{1}$, Luana Aparecida Alves da Silva ${ }^{2}$, Tatiana Brusamarello ${ }^{3}$,
\end{abstract} Edinaldo Silva de Oliveira ${ }^{4}$, Tatiane dos Santos $^{5}$, Letícia Pontes ${ }^{6}$

\section{RESUMO:}

Objetivo: Estudo de revisão integrativa que objetivou buscar evidências científicas que abordassem os principais erros de medicação e suas causas observados pela enfermagem nas instituições hospitalares de internação integral, bem como, descrever as estratégias utilizadas para promover a segurança medicamentosa nestas instituições. Método: Procedeu-se busca nas bases de dados: Literatura Latino-Americana e do Caribe em Ciências da Saúde, Base de Dados da Enfermagem e National Library of Medicine, utilizando os descritores Nursing; Medication Errors; Patient Safety; Medication Systems, Hospital. Foram selecionados 79 artigos entre os anos de 2004 a 2015. Resultados: Os tipos de erros mais citados foram dose imprópria 35 (13,3\%), omissão de dose 30 (11,5\%), e horário errado 29 (11,1\%). As causas mais citadas foram as relacionadas ao fator humano $41(34,2 \%)$, sistema $37(30,9 \%)$ e comunicação $22(18,3 \%)$. As estratégias que se destacaram foram implantação de protocolos de segurança de preparo e administração de medicamentos, prescrição eletrônica, inclusão do farmacêutico na equipe e treinamento/orientação da equipe. Conclusão: Acredita-se que esta revisão possa contribuir para melhoria do processo de administração de medicamentos e para criação de estratégias de prevenção que fomentem a segurança do paciente.

Descritores: Enfermagem; Erros de medicação; Segurança do paciente.

\footnotetext{
1 Enfermeira. Mestre em Enfermagem. Enfermeira do Complexo Hospital de Clínicas da Universidade Federal do Paraná. Curitiba-PR-Brasil.

2 Enfermeira. Especialista em Gestão e Auditoria em Saúde. Enfermeira do Complexo Hospital de Clínicas da Universidade Federal do Paraná. Curitiba-PR-Brasil.

3 Enfermeira. Doutora em Enfermagem. Enfermeira do Complexo Hospital de Clínicas da Universidade Federal do Paraná. Curitiba-PR-Brasil.

4 Enfermeiro. Mestre em Enfermagem. Enfermeiro do Complexo Hospital de Clínicas da Universidade Federal do Paraná. Curitiba-PR-Brasil.

5 Enfermeira. Mestranda em Enfermagem. Enfermeira do Complexo Hospital de Clínicas da Universidade Federal do Paraná. Curitiba-PR-Brasil.

6 Enfermeira. Doutora em Enfermagem. Professor Adjunto do Departamento de Enfermagem da Universidade Federal do Paraná. Curitiba-PR-Brasil.
} 


\begin{abstract}
Objective: Integrative review study that aimed to search for scientific evidences on major medication errors and their causes observed by nursing in in-patient facilities of hospital institutions, as well as to describe the strategies used to promote medication safety in such institutions. Method: The research was proceeded in the databases: Literatura LatinoAmericana e do Caribe em Ciências da Saúde, Base de Dados da Enfermagem and National Library of Medicine, using the descriptors Nursing; Medication Errors; Patient Safety; Medication Systems, Hospital. Seventy-nine (79) articles were selected between 2004 and 2015. Results: The most frequently reported errors were inappropriate dosage $35(13,3 \%)$, dosage omission $30(11,5 \%)$, and wrong time $29(11.1 \%)$. The most cited causes were related to human factor $4141(34,2 \%)$, system-related factors $37(30,9 \%)$ and communication 22 $(18,3 \%)$. The strategies that stood out were the implementation of safety protocols for drug preparation and administration, electronic prescription, inclusion of the pharmaceutical in the team and team training/counselling. Conclusion: It is believed that this review may contribute to improving the medication process, as well as the creation of prevention strategies which promotes patient safety.
\end{abstract}

Descriptors: Nursing; Medication errors; Patient safety.

\title{
RESUMEN
}

Objetivo: Estudio de revisión integrativa que objetivó buscar evidencias científicas que abordasen los principales errores de medicación y sus causas observadas por la enfermería en las instituciones hospitalarias de internación integral, así como, describir las estrategias utilizadas para promover la seguridad medicamentosa en estas instituciones. Método: Se buscó en las bases de datos: Literatura Latino-Americana e do Caribe em Ciências da Saúde, Base de Dados da Enfermagem ye National Library of Medicine, utilizando los descriptores Nursing; Medication Errors; Patient Safety; Medication Systems, Hospital. Se seleccionaron 79 artículos entre los años 2004 a 2015. Resultados: Los tipos de errores más citados fueron dosis impropias 35 (13,3\%), omisión de dosis 30 (11,5\%), y horario equivocado 29 (11,1\%). Las causas más citadas fueron las relacionadas al factor humano 41 (34,2\%), sistema 37 $(30,9 \%)$ y comunicación $22(18,3 \%)$. Las estrategias que se destacaron fueron la implantación de protocolos de seguridad de preparación y administración de medicamentos, prescripción electrónica, inclusión del farmacéutico en el equipo y entrenamiento / orientación del equipo. Conclusión: Se cree que esta revisión puede contribuir a mejorar el proceso de administración de medicamentos y para la creación de estrategias de prevención que fomenten la seguridad del paciente.

Descriptores: Enfermería; Errores de medicación; Seguridad del paciente.

\section{INTRODUÇÃO}

A administração segura de medicamentos está incluída, entre outras práticas, em um movimento global em busca da segurança do paciente que envolve esforços de todo o sistema de saúde, promovendo o gerenciamento de riscos e ambiente seguro. ${ }^{1}$ 
O medicamento - como estratégia terapêutica - é utilizado para controle, tratamento e cura de doenças e muito colabora para o aumento da qualidade e, até mesmo, da expectativa de vida do paciente. ${ }^{2}$ Entretanto, não é isento de riscos quando se compreende que a cadeia medicamentosa é um sistema complexo constituído de processos interligados e interdependentes e inclui diferentes fases e diversos profissionais. ${ }^{3} \quad$ Qualquer irregularidade no processo de prescrição, transcrição, dispensação, preparo ou administração de medicamentos apresenta um risco potencial ao paciente. ${ }^{4}$

O profissional de enfermagem é o protagonista nos processos de análise de riscos para a consequente redução e prevenção de incidentes ${ }^{5}$ e sua atuação se evidencia na ponta final do processo de medicação: no preparo, na administração, na avaliação da efetividade da droga administrada e na documentação do cuidado realizado. Isso aumenta a responsabilidade desses profissionais, por representarem a última oportunidade de interceptar e evitar um erro ocorrido nas fases anteriores do processo. Assim, a equipe de enfermagem é uma das últimas barreiras de prevenção. ${ }^{6}$

O National Coordinating Council for Medication Error Reporting and
Prevention (NCCMERP) define erro de medicação (EM) como "qualquer evento prevenível que possa causar ou conduzir ao uso inapropriado de medicamento ou dano ao paciente enquanto o medicamento está sob o controle do profissional de saúde, paciente ou consumidor" 7:1 Define, ainda, dano como o "comprometimento da função física, emocional ou psicológica ou estrutura corporal e/ou dor dele resultante". $8: 6$

A frequência de EM e danos preveníveis envolvendo eventos relacionados com medicamentos tem sido uma preocupação. Um paciente é exposto a, pelo menos, um EM por dia e é possível afirmar que um quarto de todos os danos relacionados com medicamento são preveníveis, de maneira que estes erros são o tipo mais comum nos serviços de saúde, frequentemente em hospitais. ${ }^{9-10}$ Nem todo EM causa dano ao paciente; entretanto, todo erro pode causar ansiedade para a equipe e paciente e também reduzir a confiança do paciente no tratamento. ${ }^{11}$ Ainda quando não há dano, a ocorrência de erros frequentemente provoca aumento da carga de trabalho e, principalmente, dos custos envolvidos no tratamento. Portanto, a segurança no uso de medicamentos representa séria fonte de preocupação, 
tanto para os prestadores de cuidados de saúde quanto para os pacientes. ${ }^{10}$

Dessa forma, identificar a natureza e os fatores determinantes dos erros envolvendo medicamentos torna-se relevante quando se avaliam as possibilidades de prevenção e contribuição para a segurança do paciente. ${ }^{12}$ Assim, evidencia-se a necessidade de que a equipe de enfermagem tenha uma visão ampla do sistema de medicação e de todos os processos envolvidos na cadeia de medicamentos, contribuindo para a terapêutica medicamentosa segura. ${ }^{13}$

Nesse contexto, este estudo objetiva buscar evidências científicas que abordem os principais EM - e suas causas observados pela enfermagem nas instituições hospitalares de internação integral, bem como, descrever as estratégias utilizadas para promover a segurança medicamentosa nestas instituições.

\section{MÉTODO}

Trata-se de uma revisão integrativa (RI) da literatura, para qual foram seguidas seis etapas metodológicas: I. Seleção das hipóteses ou questões da revisão; II. Demonstração da pesquisa a ser revista; III. Representação das características do estudo e suas constatações; IV. Análise das constatações; V. Interpretação dos resultados; e VI. Relato da revisão. ${ }^{14}$

$\mathrm{Na}$ primeira etapa, formularam-se as questões norteadoras, com fins de guiar o estudo: Quais os principais tipos e causas de EM relatados pela enfermagem nas instituições hospitalares de internação integral? Quais estratégias estão sendo utilizadas pelos enfermeiros para promover a segurança em relação ao uso de medicações nas instituições hospitalares de internação integral?

$\mathrm{Na}$ segunda etapa, deu-se início à busca dos estudos, que foi executada de forma eletrônica, em março de 2016, nas seguintes bases de dados: Literatura Latino-Americana e do Caribe em Ciências da Saúde (LILACS), Base de Dados da Enfermagem (BDENF) e National Library of Medicine (PubMed). Para tanto, utilizou-se a seguinte estratégia de busca, com descritores padronizados escolhidos a partir do Medical Subject Headings (MeSH): Nursing; Medication Errors; Patient Safety; Medication Systems, Hospital. Foi utilizado o operador booleano $A N D$ para a combinação entre eles.

Os critérios dos estudos para compor a revisão foram: ser artigo científico publicado na literatura nacional e internacional - no período de janeiro de 
2004 a dezembro de 2015 - que respondesse às questões de pesquisa nos idiomas inglês, português ou espanhol, disponível eletronicamente na íntegra. Foram excluídos os artigos que não atenderam ao objetivo proposto e aos critérios de seleção.

Na busca das bases de dados, foram encontrados 659 artigos, sendo: 98 na base LILACS; 63, na BDENF; e 498, na PubMed. Após a busca, foi realizada a exclusão de artigos repetidos. Em seguida, procedeu-se à leitura dos títulos e resumos para selecionar aqueles que se enquadravam no objetivo do estudo. A partir dessa seleção, realizou-se a leitura dos artigos na íntegra. Estas etapas foram executadas por dois revisores de forma independente. $\mathrm{O}$ processo de seleção dos artigos que compuseram a amostra foi baseado nos critérios do Preferred Reporting Items for Systematic Review and a Meta-Analyses, conforme (PRISMA) ${ }^{15}$ fluxograma abaixo (Figura 1).

Figura 1 - Fluxograma da seleção dos artigos científicos que compuseram a amostra da revisão integrativa. Curitiba, PR, Brasil, 2016 


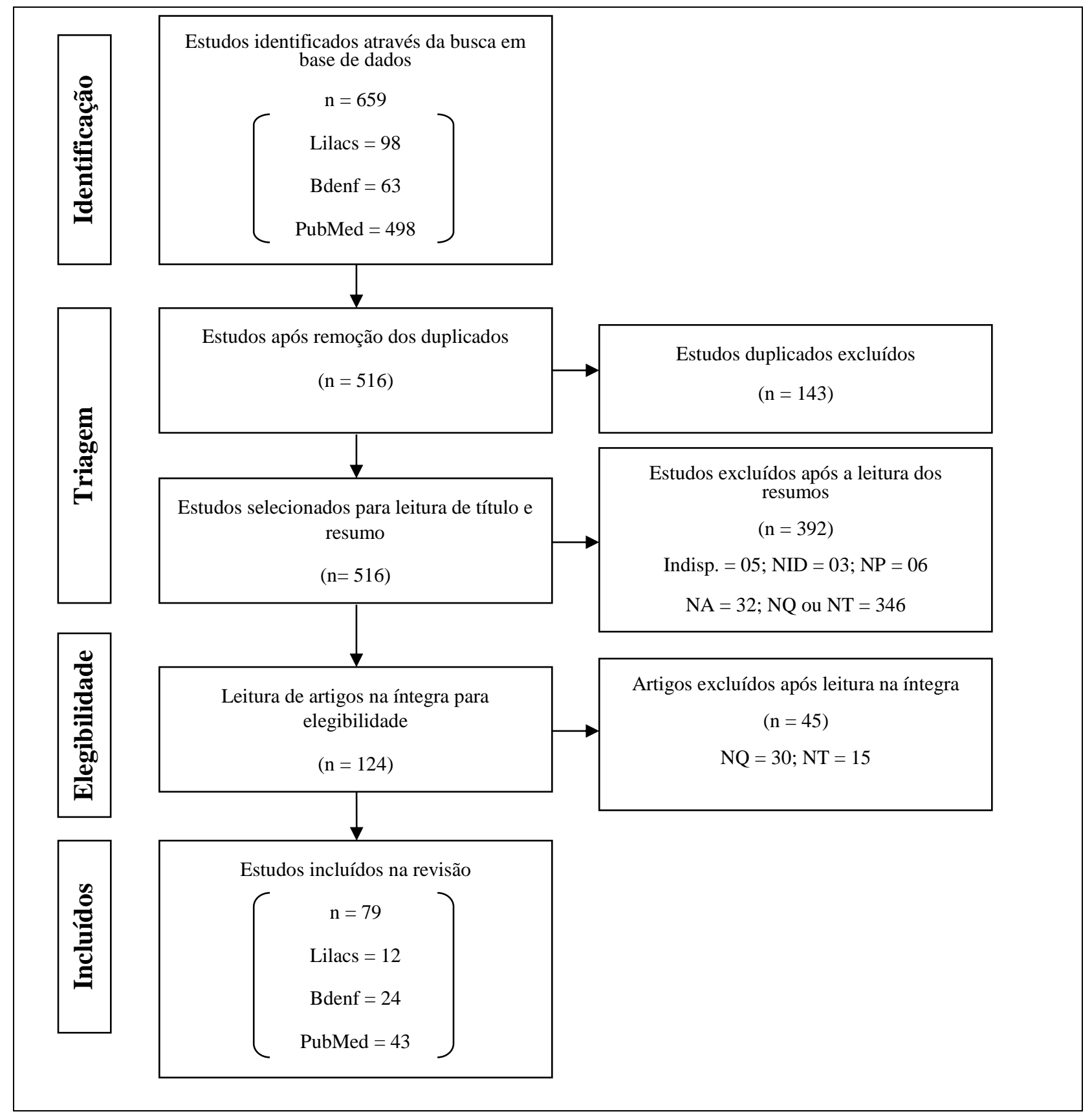

$\mathrm{NA}=$ não é artigo; $\mathrm{NP}=$ não é pesquisa; $\mathrm{NID}=$ não é do idioma; $\mathrm{NQ}=$ não responde às questões; $\mathrm{NT}=$ não é da temática; Indisp. = Artigo indisponível eletronicamente na íntegra. ${ }^{16}$

Para a execução da terceira etapa deste estudo, foi elaborado, pelos revisores, um instrumento, o qual foi preenchido no decorrer da leitura integral dos artigos, contendo: Base de dados;
Descritores; Periódico; Ano de publicação; Autores; Categoria; Idioma; Título; Objetivo; Abordagem; Tipo/Desenho do estudo; Local do estudo; Sujeitos; Tipos de 
EM; Causas de EM; Intervenção sobre o erro; Nível de evidência do estudo.

Para este último item, considerouse: Nível 1 - metanálise de múltiplos estudos controlados; Nível 2 - estudo individual com desenho experimental; Nível 3 - estudo com desenho quase experimental como estudo sem randomização com grupo único pré e pósteste, séries temporais ou caso-controle; Nível 4 - estudo com desenho não experimental como pesquisa descritiva correlacional e qualitativa ou estudos de caso; Nível 5 - relatório de casos ou dado obtido de forma sistemática, de qualidade verificável ou dados de avaliação de programas; Nível 6 - opinião de autoridades respeitáveis baseada na competência clínica ou opinião de comitês de especialistas, incluindo interpretações de informações não baseadas em pesquisas, opiniões reguladoras ou legais. ${ }^{17}$

A leitura na íntegra dos artigos e o preenchimento do instrumento de coleta de dados possibilitaram a análise dos artigos e interpretação dos resultados (quarta e quinta etapas da RI), que serão apresentadas a seguir.

\section{RESULTADOS}

Esta RI foi composta por 79 artigos. Com relação ao ano, 2011 foi o ano com mais publicações (11), seguido por 2006 (dez). Os anos de 2005, 2007 e 2010 tiveram nove publicações cada e os anos de 2012 e 2014, oito cada. Seguiu-se o ano de 2008 com quatro publicações. Os anos com menor número de publicações foram: 2004, 2009 e 2013, com três cada um; e, 2015, com dois artigos. Quanto à abordagem: 17 foram estudos qualitativos, 56 quantitativos e seis classificados como quali-quantitativos; quanto ao tipo, predominaram os estudos descritivoexploratórios.

Com relação ao local do estudo, foram selecionados apenas aqueles realizados em hospitais, com o objetivo de responder à pergunta de pesquisa. Dos 79 estudos, 39 (49\%) são nacionais e 40 $(51 \%)$ internacionais. Nos estudos internacionais, o país predominante foi Estados Unidos - com 12 (30\%) estudos seguido do Canadá, com cinco $(12,5 \%)$ e Reino Unido, com quatro (10\%). Quanto aos estudos nacionais, a maioria foi realizada nas regiões Sul e Sudeste: 28 (72\%). Os demais foram realizados nas regiões Centro-Oeste: cinco (13\%); Nordeste: três $(7,5 \%)$; e o restante - três $(7,5 \%)$ - foram estudos multicêntricos, realizados em hospitais de várias regiões do país. 
No tocante aos participantes dos estudos, 41 (52\%) foram realizados com enfermeiro e/ou equipe de enfermagem. Seis $(7,5 \%)$ tiveram a participação de médicos e/ou profissionais da farmácia concomitante com enfermeiros ou equipe de enfermagem. Houve também estudos para os quais a coleta de dados ocorreu a partir de documentações (prescrições médicas, prontuários de pacientes, sistema de notificação de incidentes) ou o objetivo do estudo foi analisar o sistema de medicação como, por exemplo, o processo de dispensação, somando 26 (33\%). Os seis $(7,5 \%)$ restantes foram estudos para os quais a coleta de dados foi composta simultaneamente por profissionais e documentação.

No quadro 1, são apresentados os tipos de erros conforme a classificação do NCCMERP. ${ }^{8}$ De acordo com os achados, $35(13,3 \%)$ estudos destacaram-se como tipo de erro na administração de medicação, como a dose imprópria; 30 (11,5\%), como omissão de dose; e 29 (11,1\%), como medicamentos administrados em horário errado. Ressaltase que alguns estudos apontaram mais de uma opção.

Quadro 1 - Erros de medicação segundo tipo. Curitiba, PR, Brasil, 2016

\begin{tabular}{|c|c|c|c|}
\hline \multirow[t]{2}{*}{ Tipos de erros de medicação } & \multirow[t]{2}{*}{ Definição } & \multicolumn{2}{|c|}{ Frequência } \\
\hline & & $\mathbf{n}$ & $\%$ \\
\hline Dose imprópria $^{1,5,18-50}$ & $\begin{array}{c}\text { Resulta em sobredosagem, subdosagem ou dose } \\
\text { extra }\end{array}$ & 35 & 13,3 \\
\hline Omissão de dose $e_{48,50,51-53,54}^{5,18,19,21,22,24,25,27-32,35-39,42-}$ & $\begin{array}{c}\text { Falha em administrar uma dose prescrita a um } \\
\text { paciente. Excluem-se pacientes que se recusam a } \\
\text { tomar a medicação }\end{array}$ & 30 & 11,5 \\
\hline $\begin{array}{l}\text { Horário errado } \\
\quad 1,18,19,21,22,24,25,27- \\
38,40,45,46,49,50,51,55-57\end{array}$ & $\begin{array}{c}\text { Administração do medicamento fora de um } \\
\text { intervalo de tempo predefinido }\end{array}$ & 29 & 11,1 \\
\hline Técnica errada ${ }_{51,54-59}^{19,21,22,25,26,29,32,33,35,36,43-45,49-}$ & $\begin{array}{l}\text { Erro de preparo de trituração/diluição de } \\
\text { medicamentos }\end{array}$ & 22 & 8,4 \\
\hline $\begin{array}{c}\text { Via de administração } \\
\operatorname{errada}^{1,18,19,22,25,26,28,29,31,32,34,36,37,40,45,46,50,51,5} \\
4,55,57,60\end{array}$ & $\begin{array}{c}\text { Administração do medicamento por via diferente } \\
\text { da pretendida }\end{array}$ & 22 & 8,4 \\
\hline $\begin{array}{c}\text { Medicamento } \\
\text { errado } 1,5,18,19,24,26,28,29,31,33,34,36- \\
38,40,41,44,45,49,51,57\end{array}$ & $\begin{array}{c}\text { Dispensação e/ou administração de medicamento } \\
\text { diferente do prescrito }\end{array}$ & 21 & 8,0 \\
\hline Velocidade de infusão errada ${ }_{33,36,44-46,49,54,57}^{19,22,23,28,29,31-}$ & $\begin{array}{c}\text { Infusão mais rápida ou mais lenta que o } \\
\text { recomendado }\end{array}$ & 15 & 5,7 \\
\hline Paciente errado $1,18,19,25,26,32,34,36,37,40,47,49,51,55$ & $\begin{array}{c}\text { Administração de medicamento ao paciente } \\
\text { errado }\end{array}$ & 14 & 5,3 \\
\hline Monitoramento errado ${ }_{59,61}^{19,26,32,36,38,43,49,53,57-}$ & $\begin{array}{l}\text { Medicamentos contraindicados, interações } \\
\text { medicamento-medicamento, interação dieta- } \\
\text { medicamento, alergias documentadas, interação } \\
\text { doença-medicamento, avaliação clínica }\end{array}$ & 12 & 4,6 \\
\hline
\end{tabular}




\begin{tabular}{|c|c|c|c|}
\hline Forma farmacêutica errada ${ }^{19,25,35,43-45,54,62}$ & $\begin{array}{l}\text { Apresentação/forma farmacêutica diferente } \\
\text { daquela prescrita }\end{array}$ & 08 & 3,0 \\
\hline Medicamento deteriorado $^{19,24,29,35,36,44,45}$ & $\begin{array}{c}\text { Dispensação e/ou administração de medicamento } \\
\text { vencido }\end{array}$ & 07 & 2,7 \\
\hline Duração errada ${ }^{32,36,39,46,54}$ & $\begin{array}{c}\text { Duração do tratamento por tempo maior ou menor } \\
\text { do que o recomendado }\end{array}$ & 05 & 1,9 \\
\hline Concentração errada $^{19,63}$ & $\begin{array}{c}\text { Concentração do medicamento diferente, maior } \\
\text { ou menor daquela prescrita }\end{array}$ & 02 & 0,8 \\
\hline \multirow{5}{*}{ Outros $^{\dagger}$} & Erro não especificado ${ }^{39,52,64-76}$ & 15 & 5,7 \\
\hline & Erro de prescrição ${ }^{18,25,32,43,46,52,53,58,67,68,77-79}$ & 13 & 5,0 \\
\hline & Erro de registro $^{18,28,31,38,43,48,50}$ & 07 & 2,7 \\
\hline & Erro de administração pelo paciente ${ }^{68,80,81}$ & 03 & 1,1 \\
\hline & Erro de transcrição ${ }^{73,79}$ & 02 & 0,8 \\
\hline Total & & 262 & 100 \\
\hline
\end{tabular}

A categorização da coluna "Outros" foi feita pelos autores deste estudo.

O quadro 2 apresenta as causas dos

erros de medicação, também agrupadas de

acordo com a classificação do

NCCMERP. ${ }^{8)}$ A revisão apontou que os

estudos referiram como principais causas

de erros o fator humano, com $41(34,2 \%)$ citações. Destacaram-se ainda: problemas em relação ao sistema 37 (30,9\%); e comunicação 22 (18,3\%). Ressalta-se que alguns estudos apontaram mais de uma opção.

Quadro 2 - Causas de erros de medicação. Curitiba, PR, Brasil, 2016

\begin{tabular}{|c|c|c|c|}
\hline \multirow[t]{2}{*}{ Causas de erros de medicação } & \multirow[t]{2}{*}{ Definição } & \multicolumn{2}{|c|}{ Frequência } \\
\hline & & $\mathbf{n}$ & $\%$ \\
\hline Fator humano ${ }_{51,54,55,57,60,64,65,69,69,70-73,80-89}$ & $\begin{array}{l}\text { Conhecimento ou desempenho deficientes; erro } \\
\text { de estocagem; erro de preparação ou transcrição; } \\
\text { estresse; carga de trabalho; fadiga e sono }\end{array}$ & 41 & 34,2 \\
\hline Sistema $_{51,53,55,64,65,67,70,71,74-76,80-83,87,90}^{1,90,22,25-27,30,32,34,36,37,40-43,45,48-}$ & $\begin{array}{l}\text { Iluminação; ruído; interrupções e distrações; } \\
\text { treinamento; equipe; falta de capacitação da } \\
\text { equipe; inexperiência da equipe; políticas e } \\
\text { procedimentos }\end{array}$ & 37 & 30,9 \\
\hline Comunicação ${ }_{53,60,67,70,71,73,80-84}^{1,5,19,22,25,26,39,46,49,51-}$ & $\begin{array}{l}\text { Falha na comunicação verbal ou escrita; falha na } \\
\text { interpretação da prescrição }\end{array}$ & 22 & 18,3 \\
\hline Embalagem, Acondicionamento $^{19,49,72,86}$ & $\begin{array}{l}\text { Embalagem inapropriada; confusão na forma } \\
\text { farmacêutica; cores e formas similares; mau } \\
\text { funcionamento de aparelhos e medidores }\end{array}$ & 04 & 3,3 \\
\hline Confusão de nomes ${ }^{19,25,51}$ & $\begin{array}{c}\text { Nomes comerciais ou químicos com escrita ou } \\
\text { sons semelhantes }\end{array}$ & 03 & 2,5 \\
\hline Etiquetagem ou Rotulagem $^{19,28,82}$ & $\begin{array}{l}\text { Rótulos similares ou etiquetas incompletas ou } \\
\text { com informações erradas sobre o produto; } \\
\text { símbolos que causem distração; falta de etiqueta } \\
\text { e/ou rótulo }\end{array}$ & 03 & 2,5 \\
\hline Outrost! $t_{t}^{18,25,27,30,32,34,37,43,50,68}$ & Fatores relacionados ao paciente & 10 & 8,3 \\
\hline Total & & 120 & 100 \\
\hline
\end{tabular}

† A categorização da coluna "Outros" foi feita pelos autores deste estudo. 
Quanto ao item intervenção sobre o erro, 27 estudos não apresentaram qualquer intervenção; 22 apresentaram apenas sugestões; outros 22 destacaram ações implementadas; e oito apresentaram as intervenções implementadas concomitante com novas sugestões de melhorias. Os resultados encontrados foram classificados quanto a intervenções relacionadas ao sistema e ao fator humano. Tanto no quesito "ação implementada" quanto "sugerida", destacaram-se os protocolos de segurança de preparo e administração de medicamentos, bem como o treinamento e/ou orientação da equipe.

Quadro 3 - Intervenções frente aos erros de medicação. Curitiba, PR, Brasil, 2016

\begin{tabular}{|c|c|c|c|c|}
\hline & & & Fre & ncia \\
\hline & & & $\mathbf{n}$ & $\%$ \\
\hline \multirow{16}{*}{ 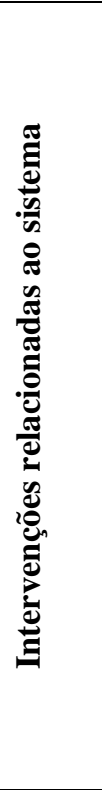 } & \multirow{10}{*}{ 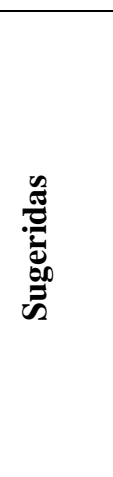 } & $\begin{array}{c}\text { Protocolos de segurança de preparo e administração de } \\
\text { medicamentos }\end{array}$ & 8 & 6,7 \\
\hline & & Prescrição eletrônica ${ }^{1,25,52,53,67,91}$ & 6 & 5 \\
\hline & & Dimensionamento de pessoal $^{1,49,50,91}$ & 4 & 3,3 \\
\hline & & Diminuição da carga de trabalho $^{49,50,55,91}$ & 4 & 3,3 \\
\hline & & Instituição de sistema de dispensação por dose unitária ${ }^{25,53,83,91}$ & 4 & 3,3 \\
\hline & & Sistema de identificação de medicamentos por código de barras ${ }^{25,35,53,64}$ & 4 & 3,3 \\
\hline & & Presença ativa do farmacêutico nas unidades ${ }^{25,52,80}$ & 3 & 2,5 \\
\hline & & Uso de pulseiras para identificação de pacientes ${ }^{25,52,67}$ & 3 & 2,5 \\
\hline & & Melhoria do ambiente de trabalho ${ }^{49,67}$ & 2 & 1,7 \\
\hline & & Modificar o processo de gestão de medicamentos $^{72}$ & 1 & 0,8 \\
\hline & \multirow{6}{*}{ 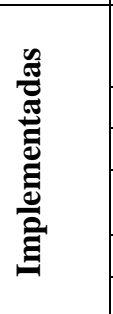 } & $\begin{array}{c}\text { Protocolos de segurança de preparo e administração de } \\
\text { medicamentos }\end{array}$ & 5 & 4,3 \\
\hline & & Inclusão do farmacêutico na equipe $\mathrm{e}^{46,49,59,74}$ & 4 & 3,3 \\
\hline & & Prescrição eletrônica $^{64,78,85,93}$ & 4 & 3,3 \\
\hline & & Investimento em equipamento eletrônico para detecção de erro $^{33}$ & 1 & 0,8 \\
\hline & & Modificação do processo de gestão de medicamentos ${ }^{75}$ & 1 & 0,8 \\
\hline & & Preparo dos medicamentos na farmácia ${ }^{93}$ & 1 & 0,8 \\
\hline \multirow{11}{*}{ 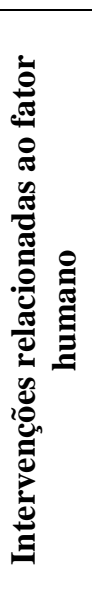 } & \multirow{9}{*}{ 㺼 } & Treinamento da equipe ${ }^{5,25,35,45,49,51-53,55,57,58,62,64,67,71,73,86,91}$ & 18 & 15 \\
\hline & & Presença efetiva/maior participação do enfermeiro $^{1,25,50,57,67,91}$ & 6 & 5 \\
\hline & & Incentivo à notificação de incidentes ${ }^{19,39,45,79,84}$ & 5 & 4,3 \\
\hline & & $\begin{array}{c}\text { Melhora na comunicação entre os elos: médico, farmácia e } \\
\text { enfermagem }\end{array}$ & 5 & 4,3 \\
\hline & & Comunicação efetiva e eficaz com o paciente p $^{5,52}$ & 2 & 1,7 \\
\hline & & Educação em saúde para o paciente ${ }^{81}$ & 1 & 0,8 \\
\hline & & Evitar estoque de medicamentos na unidade ${ }^{25}$ & 1 & 0,8 \\
\hline & & Melhoria na formação acadêmica dos profissionais em farmacologia ${ }^{49}$ & 1 & 0,8 \\
\hline & & Orientação em serviço/advertência ${ }^{51}$ & 1 & 0,8 \\
\hline & \multirow{2}{*}{$\overline{\bar{\Xi}} \mathrm{\Xi}$} & Treinamento/orientação da equipe ${ }^{1,26,28,38,44,48,51,53,63,69,75,83,87,88,91}$ & 15 & 12,5 \\
\hline & & Orientação em serviço/advertência ${ }^{26,51,55,83,91,94}$ & 6 & 5 \\
\hline
\end{tabular}




\begin{tabular}{|c|c|c|c|c|}
\hline \multirow{2}{*}{} & \multirow{2}{*}{$\begin{array}{c}\text { Intervenção pontual realizada pelo pesquisador em pesquisa } \\
\text { observacional }^{35,43,62}\end{array}$} & 3 & 2,5 \\
\cline { 2 - 4 } & & Retirada de estoque de medicamentos da unidade $^{87}$ & 1 & 0,8 \\
\hline Total & & 120 & 100 \\
\hline
\end{tabular}

$\mathrm{O}$ último item do instrumento de coleta de dados trata do nível de evidência dos estudos. Para este item, dois estudos foram classificados como nível 2; oito, como nível 3; 68, como nível 4; e um estudo, como nível 5. Nenhum dos estudos foi classificado como nível 1 ou 6 .

\section{DISCUSSÃO}

Considerando a investigação nas bases de dados, os anos 2011 e 2006 corresponderam aos maiores índices de publicações sobre a temática: $11(13,9 \%)$ e $10(12,6 \%)$, respectivamente. Entretanto, apesar do crescente interesse científico na segurança do paciente e EM pelas autoridades em saúde e, esta temática ser foco de muitas campanhas e eventos científicos nacionais e internacionais, os achados mostraram que, em 2015, constatou-se somente duas (2,5\%) publicações referentes ao tema, o que expõe a insuficiente produção científica sobre EM pelos enfermeiros.

Os resultados apresentados no quadro 1 demonstram que os estudos de enfermagem apresentam como principais tipos de EM os relacionados à falha humana, como: dose imprópria 35 (13,3\%); omissão de dose 30 (11,5\%); horário errado 29 (11,1\%); via de administração errada $22(8,4 \%)$; e técnica errada $22(8,4 \%)$. Os achados são corroborados por estudo documental realizado em um hospital geral de Goiás, que teve como um de seus objetivos identificar os eventos adversos relacionados a $\mathrm{EM}^{21}$ e também por estudo canadense realizado com enfermeiros, cujo objetivo foi examinar os fatores dentro do ambiente de trabalho que contribuem não somente para erros de administração de medicamentos, mas também para aumentar a gravidade destes. Este estudo também apontou que horário errado e dose imprópria se apresentam como os erros mais frequentes. ${ }^{34}$

Um estudo $^{21}$ realizado com profissionais envolvidos no processo de medicação de um hospital universitário, com o intuito de obter opiniões sobre EM, apontou que os profissionais culpam a si mesmos pelos erros, ignorando, assim, a visão de que o sistema em que estão envolvidos também apresenta falhas, tanto no ambiente físico quanto no 
organizacional, o que também pode colaborar - e muito - para a ocorrência dos erros de medicação. Este dado pode estar relacionado ao fato de a falha humana ser mais facilmente observada.

A dose imprópria do medicamento foi apontada como o principal tipo de erro: 35 (13,3\%). Dose imprópria é o que resulta em sobredosagem: quando há administração exagerada da dose do medicamento prescrito; subdosagem, quando a dose administrada foi menor que a necessária; e dose extra, quando o paciente recebe mais doses do que foi prescrito. $^{8} \quad$ Em estudo que objetivou analisar os EM notificados em um hospital universitário, utilizando 13 categorias de tipos de erros, a dose imprópria resultou no terceiro erro mais frequente. ${ }^{19} \mathrm{Um}$ estudo que investigou a presença de fatores que podem distrair o profissional de enfermagem durante o preparo e administração de medicamentos - e utilizou seis categorias de tipos de erros - também apontou a dose imprópria como terceiro erro mais frequente. $^{22}$

Em $30(11,5 \%)$ estudos, o erro de omissão foi apontado como um dos principais tipos de falhas na administração de medicação. Este erro é definido como falha em administrar uma dose prescrita a um paciente e quando não há comprovação da realização da medicação por meio da checagem da prescrição, excluindo-se dessa categoria os pacientes que se recusam a tomar a medicação. Referente a este dado, um estudo realizado em três unidades pediátricas de um hospital universitário da cidade de São Paulo que objetivou, por meio da análise de 68 prontuários, identificar EM, apontou que $75,7 \%$ das falhas apresentadas foram referentes aos erros de omissão. Nesse estudo, os autores apontaram que a omissão estava diretamente relacionada a falhas no registro do processo de atendimento. $^{31}$

Entende-se, por "horário errado", a administração do medicamento fora de um intervalo de tempo predefinido. Esta falha foi apontada por $29(11,1 \%)$ estudos como um dos principais tipos de erros na terapia medicamentosa. Estudo realizado em uma unidade de terapia intensiva com o propósito de identificar fatores que poderiam levar a equipe de enfermagem a distrair-se durante o preparo e a administração de medicamento observou a administração de 136 fármacos e constatou 43 erros, dentre os quais: omissão, velocidade de infusão, via, dose, diluição e horário. $^{22}$

Estudo documental que analisou os registros de enfermagem a fim de 
identificar os tipos de EM ressaltou que os erros de omissão e de horário não estavam "relacionados apenas a fatores intrínsecos à equipe de enfermagem, mas, também, a fatores associados à distribuição de medicamentos pela farmácia e aos

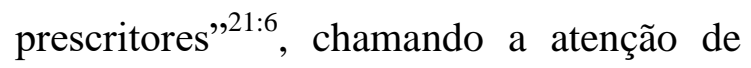
que, muitas vezes, "o determinante do erro está presente em mais de um subsistema". 21:6

Ressalte-se, neste sentido, que a enfermagem atua como a última oportunidade de interceptar e evitar um erro ocorrido nos processos iniciais da cadeia medicamentosa. Este fato aumenta a responsabilidade desses profissionais, transformando-os em uma das últimas barreiras de prevenção e garantia da segurança do paciente.

Diante dos achados deste estudo em relação aos tipos de erros, acredita-se que a implementação da prática de verificação dos certos da terapia medicamentosa medicamento certo, dose certa, via certa, horário certo, paciente certo, anotação certa, ação certa, forma certa e resposta certa - pode contribuir para enfermagem avançar na garantia de segurança ao paciente. $^{12}$

Entre as principais causas de erros citadas nos estudos, destacam-se, conforme evidenciado no quadro 2: fator humano, 41
(34,2\%); no sistema, 37 (30,9\%); e na comunicação, 22 (18,3\%). Entende-se como "causas de fatores humanos" aqueles relacionados ao conhecimento ou desempenho deficientes, erro de estocagem, erro de preparação ou transcrição, estresse, carga de trabalho, fadiga e sono. Como abordado anteriormente, a enfermagem é elementochave na cadeia que envolve o processo de medicar e pode contribuir extensivamente na segurança do processo. Por outro lado, o contingente de pessoal insuficiente; baixos salários: o que acarreta em duplo, ou até mesmo triplo vínculo de trabalho; baixa qualidade de vida; estresse; jornadas excessivas, muitas vezes, em cenários com ambientes inseguros pela falta de insumos e materiais adequados, sendo, por vezes, necessário o improviso: todos estes fatores contribuem para a ocorrência de diferentes erros.

Nas causas relacionadas ao sistema, apontadas em 37 (30,9\%) estudos, enquadram-se situações referentes à iluminação, ruído, interrupções e distrações, treinamento, falta de capacitação ou inexperiência da equipe, políticas e procedimentos. Diversos estudos $^{1,19,65,67,90}$ apontam ainda, na maioria das unidades hospitalares, a indisponibilidade ou necessidade $\mathrm{da}$ 
adequação da área física para a execução dos processos de enfermagem relacionados à medicação, bem como melhorias nas condições de iluminação do ambiente de trabalho e ações que proporcionem diminuição do desconforto acústico. Entende-se que estes são fatores primordiais para garantir a segurança no processo de medicação.

Outra causa referenciada em 22 $(18,3 \%)$ artigos foi a comunicação. Neste estudo, entende-se esta causa como falhas na comunicação verbal ou escrita, bem como os erros de interpretação da prescrição. Estudos ${ }^{19,25,52,80,83,84}$ apresentam a lacuna que, infelizmente, ainda existe quando o tema é a comunicação entre os membros das equipes médica e de enfermagem e entre membros de uma mesma equipe, principalmente em relação ao esclarecimento de dúvidas a respeito da prescrição, da suspensão ou troca de medicamentos e horários. A segurança do paciente é aumentada quando existe a integração entre a equipe médica e de enfermagem. Para tanto, porém, é necessário que a comunicação esteja presente de forma clara e efetiva em todas etapas do tratamento medicamentoso.

Quanto às intervenções frente aos EM, apenas 30 dos 79 artigos apresentaram a implementação delas (quadro 3). As intervenções implementadas relacionadas ao sistema mais citadas foram: implantação de protocolos de segurança de preparo e administração de medicamentos, implantação de prescrição eletrônica e inclusão do profissional farmacêutico na equipe. Isso reforça o envolvimento das ações de profissionais de três diferentes áreas: médica, enfermagem e farmácia. ${ }^{6}$ Estudo que analisou a eficácia do sistema de prescrição eletrônica concluiu que essa medida isolada não é capaz de diminuir os erros; é necessário, portanto, que esteja atrelada a outras intervenções, tais como: formação, treinamento e revisão de protocolos. $^{71}$

A intervenção implementada relacionada ao fator humano mais citada foi: treinamento e/ou orientação da equipe. Estudos que utilizaram análise pré e pósintervenção de treinamento da equipe concluíram que houve melhora no desempenho e diminuição de EM. $^{63,69,77}$ Essa estratégia também foi apontada, em primeiro lugar, para a melhoria da segurança com medicamentos em estudo realizado no sul do Brasil, com técnicos de enfermagem, cujo objetivo era identificar, na opinião desses profissionais, as justificativas para a ocorrência de erros de administração de medicamentos. ${ }^{65}$ 
Por fim, ressalta-se a necessidade de os hospitais direcionarem esforços para a formação da cultura de segurança voltada ao paciente, dentro da qual todos os profissionais envolvidos no processo de medicação estejam conscientes da importância de identificação, notificação e prevenção dos EM. Além disso, os gestores precisam estar atentos à necessidade da valorização dos aspectos da formação contínua de seus profissionais para as questões farmacológicas que envolvem a assistência ao paciente internado. $^{21}$

\section{CONCLUSÃO}

Este estudo apresentou como principais tipos de EM: a dose imprópria, omissão de dose e horário errado. A revisão permitiu ainda verificar que os estudos apontam como principais causas dos EM o fator humano, fatores relacionados ao sistema e à comunicação. E, em relação ao que tem sido apontado como intervenção para prevenir os EM no ambiente hospitalar, foram observadas as

\section{REFERÊNCIAS}

1. Dias JD, Mekaro KS, Tibes CMS, ZemMascarenhas SH. The Nurses' understanding about patient safety and medication errors. REME Rev Min Enferm. 2014; 18(4):585-93. intervenções ligadas ao sistema e ao fator humano, destacando-se a implantação de protocolos de segurança de preparo e administração de medicamentos, prescrição eletrônica, inclusão do profissional farmacêutico na equipe, treinamento e orientação da equipe.

Conhecer os principais tipos e causas de erros prevalentes no hospital é fundamental para a melhoria do processo de administração de medicamentos. Acredita-se que a notificação dos incidentes relacionados ao EM pode contribuir para esta identificação, bem como para a implementação de medidas administrativas voltadas ao planejamento do sistema de medicação na instituição.

A educação permanente dos profissionais de enfermagem relacionada ao processo medicamentoso é uma estratégia de baixo custo; portanto, deve ser, cada vez mais, explorada e valorizada pelos gestores, a fim de prevenir os erros na administração da medicação, garantir a segurança do paciente e o sucesso no processo de trabalho do enfermeiro.

2. Marques LFG. Uso de medicamentos e a segurança do paciente na interface entre hospital, atenção básica e domicílio. [dissertação]. São Paulo, SP: Universidade de São Paulo; 2013.

3. Mesquita MPRL. Critérios para política de gestão em terapia antineoplásica: 
segurança do paciente e do trabalhador. [tese]. Florianópolis, SC: Universidade Federal de Santa Catarina; 2014.

4. Baptista SCF. Análise de erros nos processos de preparo e administração de medicamentos em pacientes pediátricos. [dissertação]. Rio de Janeiro, RJ: Escola Nacional de Saúde Pública Sérgio Arouca; 2014.

5. Toffoletto MC, Ruiz XR. Improving patient safety: how and why incidences occur in nursing care. Rev Esc Enferm USP. 2013; 47(3):1098-105.

6. Magalhães AMM, Moura GMSS, Pasin SS, Funcke LB, Pardal BM, Krelling A. The medication process, workload and patient safety in inpatient units. Rev Esc Enferm USP. 2015; 49(n esp):42-49. 7. National Coordinating Council for Medications Errors Reporting and Prevention. About medication errors: what is a medication error? [Internet]. [S.1.]: NCCMERP; [2016] [citado em 23 maio 2016]. Disponível em: http://www.nccmerp.org/aboutmedication-errors.

8. National Coordinating Council for Medications Errors Reporting and Prevention. NCC MERP taxonomy of medication errors [Internet]. [S.1.]:

NCCMERP; [2001] [citado em 23 maio 2016]. Disponível em:

https://www.nccmerp.org/sites/default/files /taxonomy2001-07-31.pdf

9. Kohn LT, Corrigan JM, Donaldson MS, editores. To err is human: building a safer health system. Washington, DC: National Academies Press; 2000.

10. Aspden P, Wolcott J, Bootman JL, Cronenwett LR. Preventing medication errors: quality chasm series. Washington, DC: National Academies Press; 2006. 11. Blandford A, Furniss D, Lyons I, Chumgley G, Iacovides I, Wei L, et al. Exploring the Current Landscape of Intravenous Infusion Practices and Errors (ECLIPSE): protocol for a mixedmethods observational study. BMJ Open [Internet]. 2016 [citado em 19 set 2016]; 6(3):e009777. Disponível em: https://bmjopen.bmj.com/content/bmjopen/ 6/3/e009777.full.pdf

12. Ministério da Saúde (Brasil), Conselho Nacional de Saúde. Protocolo de segurança na prescrição, uso e administração de medicamentos: anexo 03. Brasília, DF: Ministério da Saúde; 2013. 46p.

13. Matias EO. Avaliação da prática de enfermagem no processo de administração de medicamento intravenoso na pediatria. [dissertação]. Fortaleza: Universidade Federal do Ceará; 2015.

14. Ganong LH. Integrative review of nursing research. Res Nursing Health. 1987; 10(1):1-11.

15. Enhancing the QUAlity and

Transparency of health Research Network. PRISMA flow diagram. [Internet]. Oxford: University of Oxford; [2009] [citado em 22 jun 2016]. Disponível em: http://www.equator-network.org/wpcontent/uploads/2013/09/PRISMA-2009Flow-Diagram-MS-Word.doc 16. Paula CC, Padoin SMM, Galvão CM. Revisão integrativa como ferramenta para tomada de decisão na prática em saúde. In: Lacerda MR, Costenaro RGS, organizadores. Metodologias da pesquisa para a enfermagem e saúde: da teoria à prática. Porto Alegre: Moriá; 2015. p.5176.

17. Galvão CM, Sawada NO, Mendes IAC. A busca das melhores evidências. Rev Esc Enferm USP. 2003; 37(4):43-50. 18. Paranaguá TTB, Bezerra ALQ, Santos ALM, Silva AEBC. Prevalência e fatores associados aos incidentes relacionados à medicação em pacientes cirúrgicos. Rev Esc Enferm USP. 2014; 48(1):41-8. 19. Yamamoto MS, Peterlini MAS, Bohomo E. Spontaneous reporting of medication errors in pediatric university hospital. Acta Paul Enferm. 2011; 24(6):766-71. 
20. Gimenes FRE, Mota MLS, Teixeira TCA, Silva AEBC, Opitz SP, Cassiani SHB. Segurança do paciente na terapêutica medicamentosa e a influência da prescrição médica nos erros de dose. Rev Latinoam Enferm. 2010; 18(6):1-7.

21. Silva AEBC, Reis AMM, Miasso AI, Santos JO, Cassiani, SHB. Eventos adversos a medicamentos em um hospital sentinela do Estado de Goiás, Brasil. Rev Latinoam Enferm. 2011; 19(2):1-9. 22. Lemos NRF, Silva VR, Martinez MR. Fatores que predispõem à distração da equipe de enfermagem durante o preparo e a administração de medicamentos. REME Rev Min Enferm. 2012; 16(2):201-07. 23. Hoefel HHK, Zini L, Lunardi T, Santos JB, Mahmud S, Magalhães AM. Análise da administração de vancomicina nas unidades de internação cirúrgicas de um hospital universitário. Online Braz J Nurs. 2004; 3(1):1-14.

24. Nascimento CCP, Toffoletto MC, Gonçalves LA, Freitas WG, Padilha KG. Indicators of healthcare results: analysis of adverse events during hospital stays. Rev Latinoam Enferm. 2008; 16(4):1-7. 25. Teixeira TCA, Cassianni SHB. Route cause analysis: evaluation of medication errors at a university hospital. Rev Esc Enferm USP. 2010; 44(1):39-46.

26. Praxedes MFS, Telles Filho PCP. Erros e ações praticadas pela instituição hospitalar no preparo e administração de medicamentos. REME Rev Min Enferm. 2011; 15(3):406-11.

27. Bohomol E, Ramos LH. Erro de medicação: importância da notificação no gerenciamento da segurança do paciente. Rev Bras Enferm. 2007; 60(16):32-6. 28. Yamanaka TI, Pereira DG, Pedreira MLG, Peterlini MAS. Redesenho das atividades de enfermagem para redução de erros de medicação em pediatria. Rev Bras Enferm. 2007; 60(2):190-6.

29. Toffoletto MC, Padilha KG.

Consequências dos erros de medicação em unidades de terapia intensiva e semiintensiva. Rev Esc Enferm USP. 2006; 40(2):247-52.

30. Bohomol H, Ramos LH. Perceptions about medications errors: analysis of answers by the nursing team. Rev Latinoam Enferm. 2006; 14(6):887-92. 31. Melo LR, Pedreira MLG. Erros de medicação em pediatria: análise da documentação de enfermagem no prontuário do paciente. Rev Bras Enferm. 2005; 58(2):180-5.

32. Manias E, Williams A, Liew D, Rixon

S, Braaf S, Finch S. Effects of patient-, environment-and medication-related factors on high-alert medication incidents. Int J Qual Health Care. 2014; 26(3):30820.

33. Ohashi K, Dykes P, McIntosh K, Buckley E, Wien M, Bates DW.

Evaluation of intravenous medication errors with smart infusion pumps in an academic medical center. AMIA Annu Symp Proc. 2013; 16(n esp):1089-98. 34. Sears K, Goodman WM. Risk factors for increased severity of paediatric medication administration errors. Healthc Policy. 2012; 8(1):1-18.

35. Berdot S, Sabatier B, Gillaizeau F, Caruba T, Prognon P, Durieux P.

Evaluation of drug administration errors in a teaching hospital. BMC Health Serv Res. 2012; 12(60):1-8.

36. Piazza G, Nguyen TN, Cios D, Labreche M, Hohlfelder B, Fanikos J, et al. Anticoagulation-associated adverse drug events. Am J Med. 2011; 124(12):1136-42. 37. Chang Y, Mark B. Moderating effects of learning climate on the impact of $\mathrm{RN}$ staffing on medication errors. Nurs Res. 2011; 60(1):32-39.

38. Sullivan MM, O'Brien CR, Gitelman SE, Shapiro SE, Rushakoff RJ. Impact of an interactive online nursing educational module on insulin errors in hospitalized pediatric patients. Diabetes Care. 2010; 33(8):1744-46. 
39. Fahimi F, Nazari MA, Abrishami R, Sistanizad M, Mazidi T, Faghihi T, et al. Transcription errors observed in a teaching hospital. Arch Iranian Med. 2009; 12(2):173-75.

40. Mark BA, Belyea M. Nurse staffing and medication errros: cross-sectional or longitudinal relationships? Res Nurs Health. 2009; 32(1):18-30. 41. Wilkins K, Shields M. Correlates of medication error in hospitals. Health Rep. 2008; 19(2):82-103.

42. FitzHenry F, Peterson JF, Arrieta M, Waitman LR, Schildcrout JS, Miller RA. Medication administration discrepancies persist despite electronic ordering. J Am Med Inform Assoc. 2007; 14(6):756-64. 43. Haw C, Stubbs J, Dickens G. An observational study of medication administration errors in old-age psychiatric inpatients. Int J Qual Health Care. 2007; 19(4):210-16.

44. Franklin BD, O'Grady K, Parr J, Walton I. Using the internet to deliver education on drug safety. Qual Saf Health Care. 2006; 15(5):329-33.

45. Prot S, Fontan JE, Alberti C, Bourdon O, Farnoux C, Macher MA, et al. Drug administration errors and their determinants in pediatric in-patients. Int $\mathbf{J}$ Qual Health Care. 2005; 17(5):381-89. 46. Simpson JH, Lynch R, Grant J, Alroomi L. Reducing medication errors in the neonatal intensive care unit. Arch Dis Child Fetal Neonatal. 2004; 89(6):480-2. 47. Nuckols TK, Bell DS, Liu H, Paddock SM, Hilborne LH. Rates and types of events reported to established incident reporting systems in two US hospitals. Qual Saf Health Care. 2007; 16(3):164-8. 48. Novak LL, Anders S, Gadd CS, Lorenzi NM. Mediation of adoption and use: a key strategy for mitigating unintended consequences of health IT implementation. J Am Med Inform Assoc. 2012; 19(6):1043-9.
49. Bagheri-Nesami M, Esmaeli R, Tajari $M$. Intravenous medication administration errors and their causes in cardiac critical care units in Iran. Mater Sociomed. 2015; 27(6):442-6.

50. Feleke AS, Mulatu MA, Yesmaw YS. Medication administration error: magnitude and associated factors among nurses in Ethiopia. BMC Nurs. 2015; 14(53):1-8.

51. Franco JN, Ribeiro G, D'Innocenzo M, Barros PBA. Percepção da equipe de enfermagem sobre fatores causais de erros na administração de medicamentos. Rev Bras Enferm. 2010; 63(6):927-32. 52. Silva AEBC, Cassiani SHB, Miasso AI, Opitz SP. Problemas na comunicação: uma possível causa de erros de medicação. Acta Paul Enferm. 2007; 20(3):272-6. 53. Oliveira RC, Camargo AEB, Cassiani SHB. Estratégias para prevenção de erros de medicação no setor de emergência. Rev Bras Enferm. 2005; 58(4):399-404. 54. Turple J, MacKinnon NJ, Davis B. Frequency and type of medication discrepancies in one tertiary care hospital. Healthc Q. 2006; 9(n esp):119-23. 55. Freitas DF, Oda JY. Avaliação dos fatores de risco relacionados às falhas durante a administração de medicamentos. Arq Ciências Saúde Unipar. 2009; 12(3):231-7.

56. Rodrigues MCS, Oliveira LC. Erros na administração de antibióticos em unidade de terapia intensiva de hospital de ensino. Rev Eletrônica Enferm. 2010; 12(3):511-9. 57. Westbrook JI, Rob MI, Woods A, Parry D. Errors in the administration of intravenous medications in hospital and the role of correct procedures and nurse experience. Qual Saf Health Care. 2011; 20(12):1027-34.

58. Renovato RD, Carvalho PD, Rocha RSA. Investigação da técnica de administração de medicamentos por sondas enterais em hospital geral. Rev enferm UERJ. 2010; 18(2):173-8. 
59. Bemt PMLA van Den, Cusell MB, Overbeeke PW, Trommelen M, Dooren D van, Ophorst WR, et al. Quality improvement of oral medication administration in patients with enteral feeding tubes. Qual Saf Health Care. 2006; 15(1):44-7.

60. Gimenes FRE, Marques TC, Teixeira TCA, Mota MLS, Silva AEBC, Cassiani SHB. Medication wrong-route administrations in relation to medical prescriptions. Rev Latinoam Enferm. 2011; 19(1):1-7.

61. Okuno MFP, Cintra RS, VanciniCampanharo CR, Batista REA. Interação medicamentosa no serviço de emergência. Einstein. 2013; 11(4):462-6.

62. Lisboa CD, Silva LD, Matos GC. Investigação da técnica de preparo de medicamentos para administração por cateteres pela enfermagem na terapia intensiva. Rev Esc Enferm USP. 2013; 47(1):53-60.

63. Simonsen BO, Daehlin GK, Johansson I, Farup PG. Improvement of drug dose calculations by classroom teaching or elearning: a randomized controlled trial in nurses. BMJ Open. 2014; 4(n esp):1-8. 64. Oliveira RB, Melo ECP. O sistema de medicação em um hospital especializado no município do Rio de Janeiro. Esc Anna Nery Rev Enferm. 2011; 15(3):480-9. 65. Lopes BC, Vargas MAO, Azeredo NSG, Behenck, A. Erros de medicação realizados pelo técnico de enfermagem na UTI: contextualização da problemática. Enferm Foco. 2012; 3(1):16-21. 66. Pelliciotti JSS, Kimura M. Medications errors and health-related quality of life of nursing professionals in intensive care units. Rev Latinoam Enferm. 2010; 18(6):1062-9.

67. Miasso AI, Silva AEBC, Cassiani SHB, Bortoli SH, Grou CR, Oliveira RC, et al. O processo de preparo e administração de medicamentos: identificação de problemas para propor melhorias e prevenir erros de medicação. Rev Latinoam Enferm. 2006; 14(3):35463.

68. Taber DJ, Spivey JR, Tsurutis VM, Pilch NA, Meadows HB, Fleming JN, et al. Clinical and economic outcomes associated with medication errors in kidney transplantation. Clin J am Soc Nephrol. 2014; 9(5):960-6.

69. Abbasinazari M, Zareh-Toranposhti S, Hassani A, Sistanizad M, Azizian H, Panahi Y. The effect of information provision on reduction of errors in intravenous drug preparation and administration by nurses in ICU and surgical wards. Acta Med Iran. 2012; 50(11):771-7.

70. Lawton R, Carruthers S, Gardner P, Wright J, McEachan RRC. Identifying the latent failures underpinning medication administration errors: an exploratory study. Health Serv Res. 2012; 47(4):1437-59. 71. Redwood S, Rajakumar A, Hodson J, Coleman JJ. Does the implementation of an electronic prescribing system create unintended medication errors? A study of the sociotechnical context through the analysis of reported medication incidents. BMC Med Inform Decis Mak. 2011; 11(29):1-11.

72. Faye H, Rivera-Rodriguez AJ, Karsh BT, Hundt AS, Baker C, Carayon P. Involving intensive care unit nurses in a proactive risk assessment of the medication management process. Jt Comm J Qual Patient Saf. 2010; 36(8):376-84.

73. Armutlu M, Foley ML, Surette J, Belzile E, McCusker J. Survey of nursing perceptions of medication administration practices, perceived sources of errors and reporting behaviours. Healthc Q. 2008; 11(n esp):58-65.

74. Myotoku M, Iwamoto C, Tomida Y, Murayama Y, Irishio K, Nakanishi A, et al. Introduction and evaluation of a newly established holiday work system in the pharmacy ward at Municipal Ikeda 
Hospital. Yakugaku Zasshi. 2006; 126(10):1003-10.

75. Amadei JL, Ferreira RCA, Francisco

CG, Brose A. Análise das solicitações de urgência no processo de distribuição de medicamentos pela farmácia hospitalar. Rev Adm Saúde. 2009; 11(45):155-60. 76. Guedes RMA, Lima FPA, Assunção AA. O programa de qualidade no setor hospitalar e as atividades reais da enfermagem: o caso da medicação. Ciênc Saúde Colet. 2005; 14(4):1065-74. 77. Gazarian M, Graudins LV. Long-term reduction in adverse drug events: an evidence-based improvement model. Pediatrics. 2012; 129(5):1334-42. 78. Franklin BD, O'Grady K, Donyai P, Jacklin A, Barber N. The impact of a closed-loop electronic prescribing and administration system on prescribing errors, administration errors and staff time: a before-and-after study. Qual Saf Health Care. 2007; 16(4):279-84.

79. Villegas AC, Herrera MCL, Franco MG, Goya ILH, Soler AV. Errores en la prescripción y transcripción de medicación en una unidad neonatal. An Pediatr. 2006; 64(4):330-5.

80. Marques LFG, Romano-Lieber NS. Segurança do paciente no uso de medicamentos após a alta hospitalar: estudo exploratório. Saúde Soc. 2014; 23(4):1431-44.

81. Miasso AI, Cassiani SHB.

Administração de medicamentos: orientação final de enfermagem para a alta hospitalar. Rev Esc Enferm USP. 2005; 39(2):136-44.

82. Azevedo O, Silva CMB, Araújo LJDP, Costa EO, Fernandes MICD, Lira ALBC. Dificuldades vivenciadas por técnicos de enfermagem no preparo de medicamentos. Rev Rene. 2014; 15(4):585-93.

83. Cassiani SHB, Teixeira TCA, Opitz SP, Linhares JC. O sistema de medicação nos hospitais e sua avaliação por um grupo de profissionais. Rev Esc Enferm USP. 2005; 39(3):280-7.

84. Corbellini VL, Schilling MCL, Frantz SF, Godinho TG, Urbanetto JS. Eventos adversos relacionados a medicamentos: percepção de técnicos e auxiliares de enfermagem. Rev Bras Enferm. 2011; 64(2):241-7.

85. Simonsen BO, Johansson I, Daehlin GK, Osvik LM, Farup PG. Medication knowledge, certainty, and risk of errors in health care: a cross-sectional study. BMC Health Serv Res. 2011; 11(175):1-9.

86. Morgan N, Luo X, Fortner C, Frush K. Opportunities for performance improvement in relation to medication administration during pediatric stabilization. Qual Saf Health Care. 2006; 15(3):170-83.

87. Marck PB, Kwan JA, Preville B, Reynes M, Morgan-Eckley W, Versluys R, et al. Building safer systems by ecological design: using restoration science to develop a medication safety intervention. Qual Saf Health Care. 2006; 15(2):92-7. 88. Nelson NC, Evans RS, Samore MH, Gardner RM. Detection and prevention of medication errors using real-time bedside nurse charting. J Am Med Inform Assoc. 2005; 12(4):390-7.

89. Raduenz AC, Hoffmann P, Radunz V, Dal Sasso GT, Maliska IC, Marck PB. Nursing care and patient safety: visualizing medication organization, storage and distribution with photographic research methods. Rev Latinoam Enferm. 2010; 18(6):1045-54.

90. Oliveira RC, Cassiani SHB.

Characterization of the structure for medication preparation in teachinghospitals: factors that interfere with the quality of care. Rev Latinoam Enferm. 2007; 15(2):224-9.

91. Silva BK, Silva JS, Gobbo AFF, Miasso AI. Erros de medicação: condutas e propostas de prevenção na perspectiva da 
equipe de enfermagem. Rev Eletrônica Enferm. 2007; 9(3):712-23.

92. Cohen MM, Kimmel NL, Benage MK, Cox MJ, Sanders N, Spence D, et al. Medication safety program reduces adverse drug events in a community hospital. Qual Saf Health Care. 2005; 14(3):169-74.

93. Apkon M, Leonard J, Probst L, DeLizio L, Vitale R. Design of a safer approach to intravenous drug infusions: failure mode effects analysis. Qual Saf Health Care. 2004; 13(4):265-71.

94. Santos AE, Padilha KG. Eventos adversos com medicação em serviços de emergência: condutas profissionais e sentimentos vivenciados por enfermeiros. Rev Bras Enferm. 2005; 58(4):429-33.

RECEBIDO: 23/12/17

APROVADO: $13 / 06 / 18$

PUBLICADO: 09/18 